\title{
¿Qué método utilizar para estimar la temperatura estática de una
}

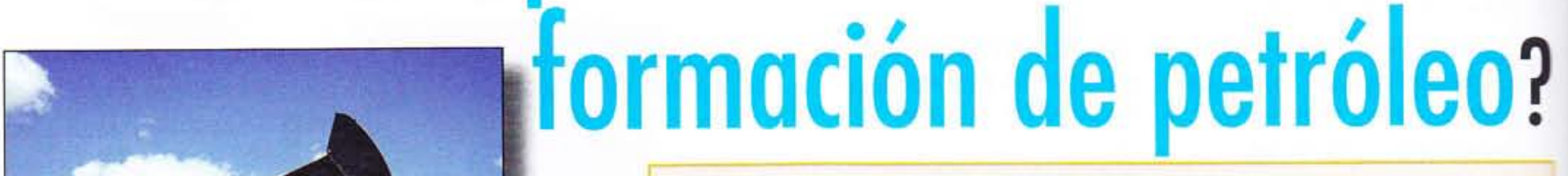

Javier Rodríguez-Martin Ignacio López-Paniagua Ângel Jiménez-Álvaro

Rafael Nieto-Carlier

* ETSI Industriales, (/ Jose Gutiérrez Abascal, 2 - 28006 Madrid. Tfno: +34 913364263. javier.rodriguez.martin@upm.es; ilopez@etsii.upm.es; rnieto@etsii.upm.es; a.jimenez@upm.es

Recibido: 27/07/2010 • Aceptado: 11/12/2010

\section{Which method to use for estimating the static formation temperature of a petroleum reservoir?}

\section{ABSTRACT}

- The static formation temperature of a petroleum reservoir is relevant for evaluating its properties. There is a variety of methods for estimating it. However, each method assumes different hypotheses and simplifications which lead to diverging estimations, in some cases far from the real value. This makes difficult knowing in advance which method to use. In this paper, the most common methods (Horner, spheric and radial, two-measures and cylindric source) are applied to four different wells. The way of applying them to real cases is described, paying special attention to the necessary input data in each case: especially thermophysical properties and number of measurements. Criteria for estimating properties in case their real value cannot be known are also provided. The paper concludes suggesting a decision tree for selecting the most appropriate method depending on the available information.

- Keywords: petroleum, static formation temperature, reservoir, estimation methods, Horner.

\section{RESUMEN}

Conocer la temperatura estática de una formación de petróleo es importante a la hora de evaluar y terminar un pozo. Existe una gran variedad de métodos para la determinación de esta temperatura. Cada método utiliza hipótesis y simplificaciones distintas que llevan a estimaciones diferentes, en algunos casos bastante alejadas del valor real. Esto hace dificil saber qué método utilizar. En este trabajo, se aplican los métodos de cálculo más comunes Horner (HM), flujo radial y esférico (SRM), de las dos medidas (TLM) y de fuente de calor cilíndrica (CSM)a cuatro pozos distintos. Se describe cómo aplicarlos en casos reales. Se presta especial atención a establecer los datos necesarios en cada caso: propiedades termo-físicas y número de medidas, y se proporcionan criterios para estimarlos en caso de no conocer su valor real. Como conclusiones a este trabajo se presentan una serie de pautas que permiten seleccionar el método de cálculo más conveniente en función de la información de que se disponga.
Palabras clave: petróleo, temperatura de estática, formación, métodos de cálculo, Horner.

\section{INTRODUCCIÓN}

Las empresas explotadoras de yacimientos de petróleo diseñan y construyen sus instalaciones optimizándolas para el tipo de petróleo que contiene cada reservorio y para el volumen de producción que se puede obtener de él. Por ello, tener información precisa sobre ambos aspectos lo antes posible puede permitir ahorros importantes en las inversiones en infraestructuras y también acortar los plazos de comienzo de explotación; ambos, aspectos esenciales en la rentabilidad del yacimiento.

La determinación del volumen de producción esperable exige el cálculo del "petróleo original in-situ" (Bidner, 2001). Este cálculo depende de cuatro factores: el volumen de roca del reservorio, la porosidad de esa roca, porcentaje de los poros ocupados por agua y el factor de volumen de petróleo. Los tres primeros se estiman mediante el análisis de muestras 
das en la perforación. El factor de volumen de leo representa la relación entre el volumen de petróleo ndiciones de reservorio (presión y temperatura) y en ciones estándares de superficie. Se determina mediante laciones que dependen de la presión a la que se encuentra ervorio y de su temperatura de formación. Para obtener letalle, consultar Bidner (2001).

sto hace que la temperatura y la presión del reservorio dos características especialmente representativas del o. La manera ideal de medirlas sería en el momento erforar el pozo, introduciendo sondas de presión mperatura, aprovechando que éste aún no está en tación. Normalmente se hace así, sin embargo las das obtenidas no son las buscadas, especialmente en el de la temperatura. La razón es que el propio proceso rforación perturba el estado del reservorio y provoca ios en su presión y su temperatura originales (Espinosates et al., 2009).

os pozos se perforan utilizando taladros que van ando el terreno hasta alcanzar el reservorio. Este so requiere refrigerar el taladro y extraer la tierra rada, para lo que se inyecta por el agujero un fluido que nasta el taladro, lo refrigera y sale otra vez a la superficie rando los residuos de tierra y rocas. Este fluido se suele r fluido de circulación. En el momento en que el taladro ra finalmente en el reservorio, cae dentro cierta cantidad rido de circulación con tierra y rocas. Puede transcurrir tiempo desde este momento hasta que se detecta que alcanzado el reservorio y se extrae el taladro, durante al sigue vertiéndose fluido de circulación y tierra. El ento en que se extrae el taladro marca el final de la ración del pozo, indicado en la Figura 1.

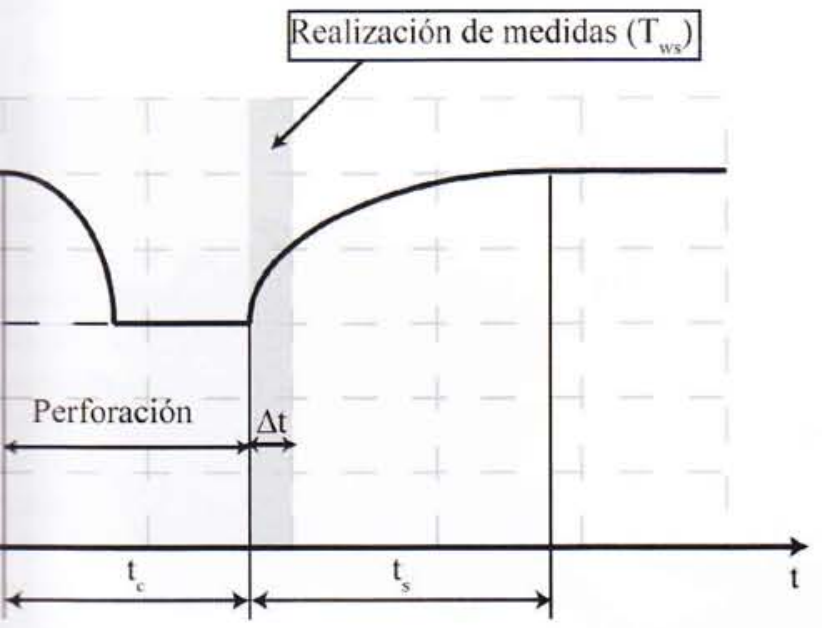

Varioción de la temperatura medida en los sensores a lo largo del tiempo

I tiempo $t_{c}$ que transcurre previamente a extraer el ro determina las condiciones de temperatura que serán tradas por las sondas. La cantidad de fluido, tierra y vertida al reservorio se encuentra a una temperatura más baja que la que tenía el reservorio antes de la perforación, por lo que, al entrar en contacto con el petróleo, bajará la temperatura del reservorio en las proximidades del pozo. Las sondas de temperatura, situadas precisamente en esta zona, medirán por tanto valores que no son los característicos del reservorio. Sin embargo, como la masa de petróleo en el reservorio es mucho más grande que la masa del vertido, la temperatura medida $T_{w s}$ se irá elevando a medida que la zona perturbada recupere su temperatura original $T_{f}$. Este proceso es la evolución de la temperatura del intervalo $t_{s}$ representada en la Figura 1. Dejando una sonda de temperatura dentro del pozo durante suficiente tiempo, terminaría midiendo la temperatura original del pozo, que llamaremos temperatura estática de la formación $T_{f}$. Al tiempo necesario para que esto ocurriese, medido desde el final de la perforación, lo llamaremos tiempo de estabilización del pozo $t_{s}$

Normalmente, por razones económicas, hay que retirar las sondas antes de alcanzarse el tiempo de estabilización, por lo que las mediciones de temperatura consistirán en un conjunto de medidas $T_{w s}$ tomadas durante un intervalo de tiempo $\Delta t<t_{s}$, cuyo valor máximo es inferior a la $T_{f}$.

El conjunto de medidas de temperatura obtenidas $T_{w s}$ será una serie de valores ascendentes, pero no llegará al valor buscado. Existen diversas alternativas para proceder que son comunes en la práctica. La más elemental es utilizar el máximo valor de temperatura medido como si fuera la temperatura estática de la formación. También se puede utilizar la serie de medidas tomadas para estimar cuál sería el valor real de la temperatura estática. Existen dos familias de métodos. Los llamados indirectos se basan en reglas empíricas que describen la variación de la temperatura con el tiempo. Para estimar $T_{f}$, se sustituyen los valores medidos en las fórmulas y se ajustan los parámetros de cálculo iterativamente, hasta minimizar los errores de ajuste. Los segundos son los métodos llamados directos, que están basados en modelos de ecuaciones diferenciales que describen los fenómenos de transmisión de calor en el reservorio. Debido a la complejidad del problema y a la ausencia de datos sobre las características del reservorio, suelen simplificarlo de forma que sea resoluble y a la vez representativo de cada caso.

Este artículo va a tratar de esta segunda familia de métodos, y va a aportar criterios para elegir cuál emplear en función de los datos de partida de que se disponga en cada caso. En particular, analizaremos la aplicabilidad y exactitud de cuatro métodos muy extendidos (Santoyo, et al., 2000): Horner (HM), flujo radial y esférico (SRM), de las dos medidas (TLM) y de fuente de calor cilindrica (CSM). Para ello, los aplicaremos a cuatro pozos de características diferentes: ROUX1, HYODO1, DOWDLE1 y SHEN1.

\section{MÉTODOS DE CÁCULO}

Como se ha mencionado previamente, en este artículo vamos a analizar cuatro métodos directos para estimar la 
temperatura estática de una formación de petróleo. Antes de proceder, es necesario describirlos brevemente.

(1) Método de Horner (HM): Este método modeliza la perforación como una fuente de calor cilíndrica constante y infinitamente larga. Considera que la temperatura es uniforme en el instante inicial, que el gradiente radial de temperatura permanece constante a lo largo del tiempo y que la temperatura es constante en regiones alejadas (Dowdle \& Cobb, 1975).

(] Método del flujo radial y esférico (SRM): Este método modeliza la zona perturbada por la perforación como una región esférica, asumiendo que la formación de roca es infinita, homogénea e isotrópica con propiedades termofísicas constantes. Se desprecia la perturbación producida por la circulación de los fluidos (Ascencio, et al., 2006).

( Método de las dos medidas (TLM): Modeliza la perforación como un cilindro con una distribución inicial de temperaturas, $T_{m}$ en el eje y $T_{f}$ en las paredes, inmerso en un medio infinito a temperatura constante $T_{f}$. Utiliza la ecuación de difusividad térmica que describe la variación de temperatura en este modelo. (Kritkos \& Kutasov, 1988, Carslaw, \& Jaeger, 1959).

( Método de la fuente de calor cilíndrica (CSM): Supone un modelo físico basado en una fuente de calor cilíndrica. Se basa en los procesos transitorios de intercambio de calor que se producen entre el líquido de circhlación y la formación. Se consideran mecanismos de conducción y convección (Hasan \& Kabir, 1994).
Todos ellos tienen en común tratar el problema dividiendo en dos la región circundante a la perforación (ver Figura 2). Distinguen por un lado las regiones alejadas de ésta, que asumen que se hallan a temperatura $T_{f}$, como lo estarían antes de perforar. Por otro lado consideran la zona afectada térmicamente por los vertidos de la perforación, inmediatamente próxima a ella. En un punto de esta zona es en la que se midieron los valores de temperatura

La primera diferencia entre los métodos es la geometría que asumen para la zona perturbada. Los métodos HM, TLM y CSM, suponen que la zona perturbada es un cilindro cuyo eje coincide con el eje del pozo (ver Figura 2); las medidas estarían tomadas en un punto de ese eje. El método SRM asimila la zona perturbada a una esfera cuyo centro se sitúa en la intersección del pozo con el reservorio; las medidas estarian tomadas aproximadamente en este punto.

Aparte de las hipótesis geométricas, las diferencias principales entre ellos están en cómo modelizan el proceso de transmisión de calor en la zona perturbada. Brevemente, se puede decir que todos los métodos consideran transmisión por conducción y algunos también por convección, y que algunos consideran condiciones de contorno constantes en algunos puntos y otros consideran condiciones variables.

Como resultado de todo ello, cada método produce una fórmula basada en parámetros propios. Estos parámetros se calculan bien con la serie de medidas de temperatura disponible, bien con datos sobre las características geotérmicas del pozo a estudiar, o bien combinando ambos. Las fórmulas de cada método y la definición de los parámetros se muestran en la Tabla 1.

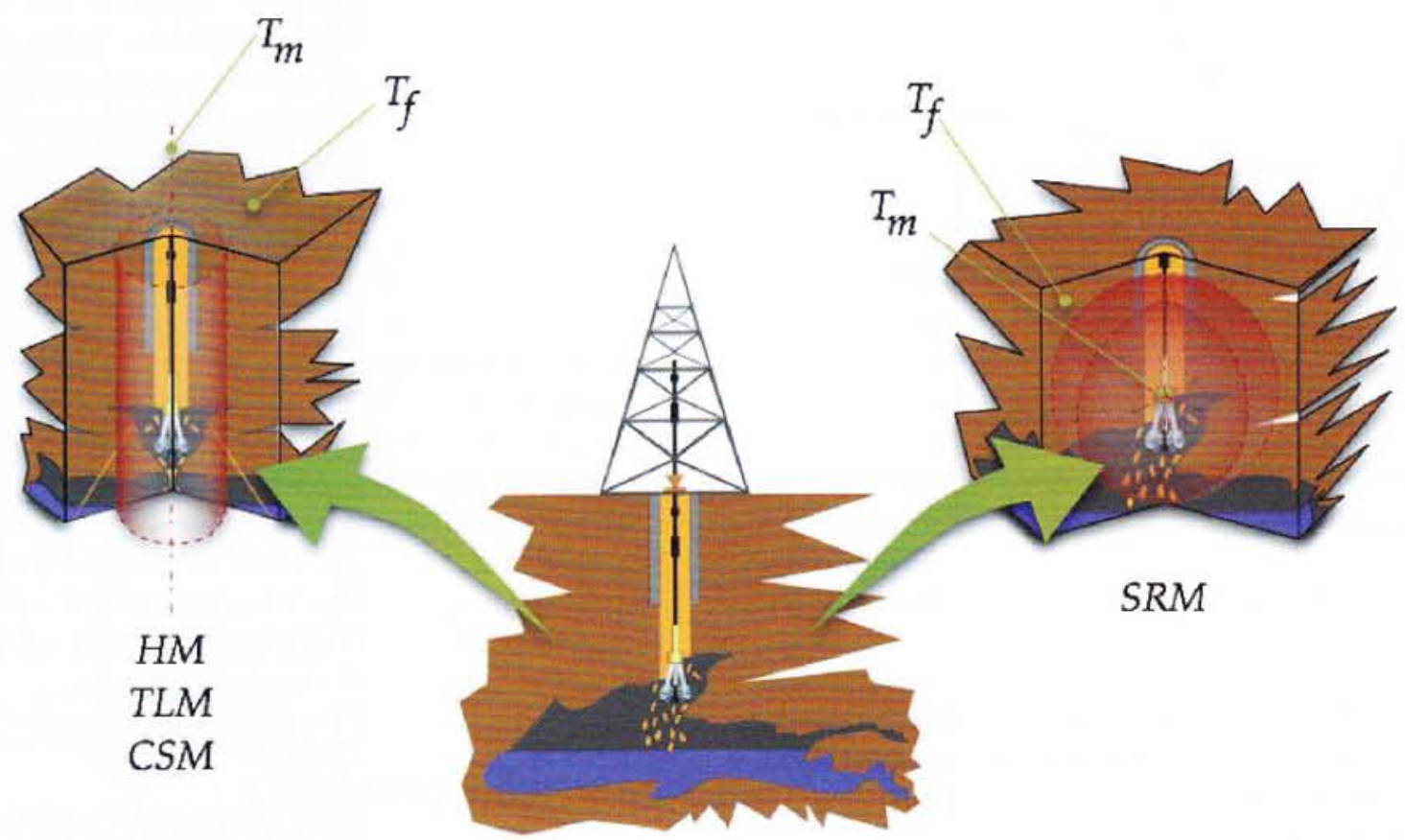

Figuro 2: Hipótesis de los distintos métodos empleados en este estudio 


\begin{tabular}{|c|c|c|}
\hline létodo & Ecuación & Aplicación \\
\hline & $T_{w s}=T_{f}+b_{H M} \cdot \log \frac{\left(t_{c}+\Delta t\right)}{\Delta t}$ & $\begin{array}{l}\text { Regresión lineal entre los valores de } \\
\log \left(\left(t_{c}+\Delta t\right) / \Delta t\right) \text { y } T_{\text {ws. }}\end{array}$ \\
\hline & $T_{w s}=T_{f}-m \cdot \frac{1}{\sqrt{\Delta t}}$ & $\begin{array}{l}\text { Regresión lineal entre los valores de } 1 / \sqrt{ } \Delta t \text { y } T_{\text {ws }} \\
\text { Válido para: } \Delta t \geq 2 \cdot r_{w}^{2} / \mathrm{a}\end{array}$ \\
\hline & $\begin{array}{c}T_{f}=T_{w s 2}+\gamma \cdot\left(T_{w s 1}-T_{w s 2}\right) \\
\gamma=\frac{E_{i}\left(-D_{1} / F_{t 2}\right)+\ln \left(F_{t 2}\right)-D_{2}}{E_{i}\left(-D_{1} / F_{t 2}\right)-E_{i}\left(-D_{1} / F_{t 1}\right)+\ln \left(F_{t 2} / F_{t 1}\right)} \\
D_{1}=1,1925 \quad D_{2}=0,7532 \\
F_{t 1}=\frac{\Delta t_{1}}{t_{c}} \quad F_{t 2}=\frac{\Delta t_{2}}{t_{c}}\end{array}$ & $\begin{array}{l}\text { Se agrupan las medidas de temperatura } T_{\text {ws }} \mathrm{y} \\
\text { tiempo } \Delta t \text { por parejas }\left(\mathrm{T}_{\text {wst }}, \Delta \mathrm{t}_{1}\right) \text { y }\left(\mathrm{T}_{\text {ws } 2}, \Delta \mathrm{t}_{2}\right) \text {. } \\
\text { Se aplica la expresión para cada pareja. } \\
\text { La temperatura estática de la formación es la media } \\
\text { de todas las temperaturas obtenidas. }\end{array}$ \\
\hline & $\begin{array}{c}T_{w s}=T_{f}-C_{0} \cdot\left|F\left(t_{D}+\Delta t_{D}\right)-F\left(t_{D}\right)\right| \\
\Delta t_{D}=\frac{\alpha \cdot \Delta t}{r_{w}^{2}} \quad t_{D}=\frac{\alpha \cdot t_{c}}{r_{w}^{2}}\end{array}$ & $\begin{array}{l}\text { Regresión lineal entre los valores de } \\
\left|F\left(t_{D}+\Delta t_{D}\right)-F\left(t \Delta t_{D}\right)\right| \text { y } T_{w s^{*}}\end{array}$ \\
\hline
\end{tabular}

1: Métodos de cálculo de la temperatura estática de la formación

En los métodos HM, SRM y CSM la temperatura tica de la formación se determina obteniendo la recta de esión entre las medidas $T_{w s}$ y una función de tiempo

$$
\left(\frac{\Delta t+t_{c}}{t_{c}}\right), \frac{1}{\sqrt{\Delta t}} \quad \text { y }\left|F\left(t_{D}+\Delta t_{D}\right)-F\left(t_{D}\right)\right|
$$

ectivamente. En el método SRM las medidas uidas en la regresión deben de ser mayores a dos veces uadrado del radio de pozo $r_{w}$ divido por la difusividad vica de la formación a . En el método CSM, la función depende de las propiedades termo-físicas del pozo, su ulo en detalle se presenta en Hasan y Kabir (1994). El odo TLM se aplica resolviendo la ecuación de la Tabla 1 cada pareja de temperaturas y calculando la media de os los resultados. La función $E_{i}$ representa a la integral onencial y se resuelve por métodos de aproximación ramowitz \& Stegun, 1964).

\section{DATOS DE MEDIDAS Y PROPIEDADES TERMO- FISICAS DE LOS POZOS}

Se van aplicar los métodos descritos en la sección erior a cuatro pozos:

ROUX1. Tiempo de circulación $t_{c}=12 h$ (Roux, et al., 1980).
4 HYODO1. Tiempo de circulación $t_{c}=5 h$ (Takai, et al., 1994).

口 DOWDLE1. Tiempo de circulación $t_{c}=3,5 \mathrm{~h}$ (Dowdle \& Cobb, 1975).

․ SHEN1. Tiempo de circulación $t_{c}=5 h$ (Shen \& Beck, 1986).

En la Figura 3 se encuentran reflejados las medidas de temperatura $T_{w s}$ y tiempo $\Delta t$ para cada uno de los cuatro pozos.

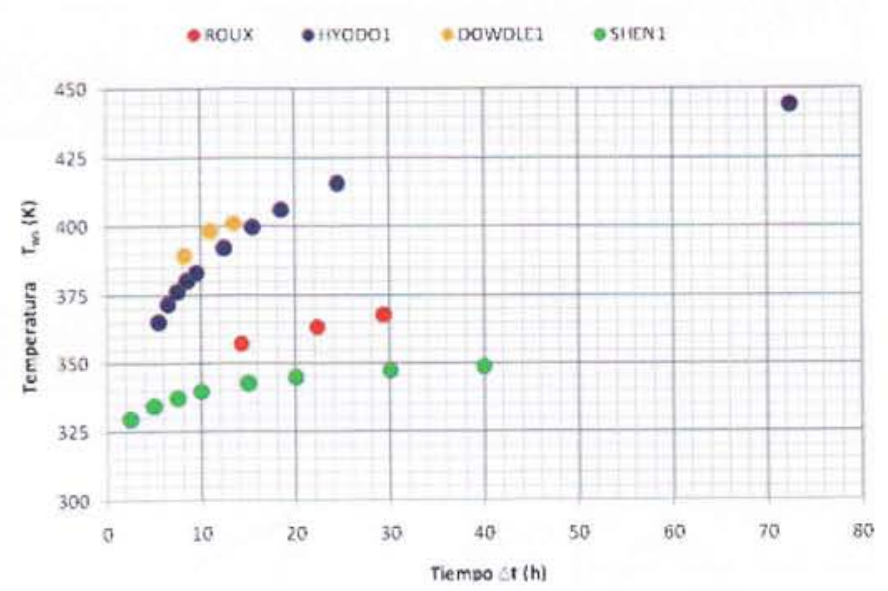

Figura 3: Temperaturas medidas y fiempos para los cuatro pozos 
En muchas ocasiones se desconocen las variables que son necesarias para estimar la temperatura estática de la formación según los diferentes métodos de cálculo. A continuación se presentan una serie de criterios que se pueden utilizar para estimar esos valores:

प Propiedades térmicas de la formación: Valores típicos de la conductividad térmica, capacidad calorífica y densidad de la formación son, respectivamente (Takai, et al.,1994).

$$
\begin{aligned}
& 0,42-4,2 \mathrm{w} / \mathrm{mK} \\
& 837,36-1004,83 \mathrm{~J} / \mathrm{kg} \mathrm{K}
\end{aligned}
$$$$
1300-3000 \mathrm{~kg} / \mathrm{m}^{3}
$$

口 Propiedades térmicas del fluido de circulación: Se pueden aproximar a las propiedades térmicas del agua (Hasan \& Kabir, 1994).

Coeficiente de transmisión de calor: Se puede aproximar al cociente entre la conductividad térmica del fluido de circulación y el radio del pozo (Hasan \& Kabir, 1994).

Difusividad térmica de la formación: Se puede calcular a partir de las propiedades térmicas de la formación o considerar un valor de $3,5 \cdot 10^{-7} \mathrm{~m}^{2} / \mathrm{s}$ (Takai, et al.,
1994)

Radio del pozo: Se puede tomar un valor tipo de 0,1 $m$ (Takai, et al.,1994).

Masa de fluido de circulación: Se calcula multiplicando la sección de perforación por la densidad del fluido de circulación.

Las propiedades termo-físicas de los cuatro pozos de estudio vienen reflejadas en la Tabla 2 (con el símbolo * vienen marcadas las que se han estimado según los criterios anteriores). Para el pozo SHEN1 son conocidas la mayoría de las propiedades termo-físicas, para los pozos ROUX1 y DOWDLE1 se conocen algunas de ellas, mientras que para el pozo HYODO1 se han tenido que estimar todas ellas.

Un parámetro que permite evaluar la rapidez de un pozo es el cociente entre la difusividad térmica y el cuadrado del radio del pozo $\left(\mathrm{a} / r_{w}{ }^{2}\right)$. En un pozo lento, como el HYODO1 considerado aquí, este cociente tiene un valor muy inferior a 0,4 (Dowdle \& Cobb, 1975).

\section{RESULTADOS}

En la Tabla 3 se aprecian los resultados obtenidos al aplicar los cuatro métodos de estimación de la temperatura estática de formación (HM, SRM, TLM y CSM) a los cuatro pozos (ROUX1, HYODO1, DOWDLE1 y SHEN1). Para

\begin{tabular}{|c|c|c|c|c|}
\hline & Roux 1 & HYODO1 & DOWDLET & SHEN1 \\
\hline Conductividad termica de la formación (W/m K) & 2,42 & $0,42^{\circ}$ & 2,42 & 2,5 \\
\hline Calor especifico del fluido de circulaction (U/kg K) & $4186,73^{\circ}$ & $4186,73^{\circ}$ & $4186,73^{\circ}$ & 3491,6 \\
\hline Difusividad térmica formación $\left(\mathrm{m}^{2} / \mathrm{h}\right)$ & 0,0037 & 0,0012 & 0,0037 & 0,0043 \\
\hline Radio del pozo $(\mathrm{m})$ & 0,117 & $0,1^{\circ}$ & $0,1^{\circ}$ & 0,108 \\
\hline Coeficiente de transmisión de calor $\left(\mathrm{W} / \mathrm{m}^{2} \mathrm{~K}\right)$ & $5,68^{\circ}$ & $14^{\circ}$ & $5,68^{\circ}$ & 5,55 \\
\hline Masa del fluido de circulación $(\mathrm{kg} / \mathrm{m})$ & $43,28^{\circ}$ & $31,42^{\circ}$ & $31,42^{\circ}$ & $43,97^{\circ}$ \\
\hline Parámetro of/r ${ }^{2}\left(\mathrm{~h}^{-1}\right)$ & 0,270 & 0,126 & 0,372 & 0,43 \\
\hline
\end{tabular}
ello se han utilizado las propiedades termo-físicas de la

Tabla 2: Propiedades termo-físicas de los pozos objeto de estudio

\begin{tabular}{|l|l|l|l|l|l|}
\cline { 2 - 6 } \multicolumn{1}{c|}{} & \multicolumn{5}{c|}{ TEMPERATURA ESTATICA DE FORMACION } \\
\hline P0ZO & Real (K) & HM (K) & SRM (K) & TLM (K) & CSM (K) \\
\hline ROUX1 & 388,15 & 380,51 & 391,28 & 378,55 & 373,92 \\
\hline HYOD01 & 489,05 & 440,45 & 483,01 & 432,74 & 434,97 \\
\hline DOWDIET & 422,04 & 423,91 & 444,34 & 421,41 & 426,35 \\
\hline SHEN1 & 353,15 & 350,04 & 358,06 & 347,69 & 351,48 \\
\hline
\end{tabular}

Tabla 3: Resultados obtenidos para la temperatura estática de la formación 


\section{CONCLUSIONES}

De acuerdo con el proceso que sufre un reservorio de petróleo al perforar que muestra la Figura 1, para poder calcular la temperatura de formación sería necesario conocer $T_{m}$ y la forma de la evolución de la temperatura con el tiempo. En la práctica, podría medirse $T_{m}$ y se mide la evolución de la temperatura durante un periodo $\Delta t$, más corto que $t_{s}$. El problema consiste en estimar los parámetros anteriores a partir de éstos. En la realidad, el instante en que se tomó la primera medida es incierto; el número de medidas disponible puede ser muy reducido ( 2 ó 3), se desconoce la desproporción entre el período de medición $\Delta t$ y el de establecimiento $t_{s}$, y el resto de parámetros necesarios (las propiedades del pozo) para reconstruir la posible evolución de la temperatura frecuentemente también son desconocidos.

El estudio presentado aquí muestra cómo influye la información disponible sobre la estimación de $T_{f}$. Disponer de valores para las características del reservorio ayuda a determinar adecuadamente en qué momento de la evolución del pozo se tomaron las medidas y la rapidez de la recuperación de la temperatura. De esta forma el intervalo $T_{f}-T_{m}$ queda fijado por las expresiones de la Tabla 1 , siendo más precisa la estimación del método que más información usa, CSM.

La Figura 5 muestra un árbol de decisión que permite optimizar la estimación de $T_{f}$ dependiendo de la información de que se disponga. En caso de disponer de pocas medidas y de no disponer de ninguna información en absoluto sobre las características del pozo, evidentemente no queda determinada la curva de evolución de la temperatura, y es necesario recurrir a aproximaciones de tipo estadístico como el método de Horner (de ahí que sea el más utilizado).

En los casos intermedios, aquellos en que se disponga de alguna información, aunque fuera cualitativa, sobre si el pozo es rápido o lento, podrían obtenerse estimaciones razonables con los otros métodos (SRM Y TLM). Este tipo de información podría deducirse en muchos casos por proximidad geográfica a otros pozos.

La conclusión fundamental es que debe explotarse al máximo la información que se tenga a priori sobre el reservorio y utilizarla ordenadamente según el árbol de decisión de la figura. Tanto la información cualitativa sobre la rapidez del pozo como estimaciones tentativas de las propiedades termo-físicas, pueden ayudar a una estimación más exacta.

\section{BIBLIOGRAFIA}

- Abramowitz M, \& Stegun IA. (1964). Handbook of mathematical functions with formulas, graphs, and mathematical tables. Dover publications.

- Andaverde J, Verma SP, \& Santoyo E. (2005). Uncertainty estimates of static formation temperatures in boreholes and evaluation of regression models. Geophysical Journal International, 160(3), 1112-1122.
- Ascencio F, Samaniego F, \&t Rivera J. (2006). Application of a spherical-radial heat transfer model to calculate geothermal gradients from measurements in deep boreholes. Geothermics, 35(1), pp 70-78.

- Bidner MS (2001). Propiedades de la roca y los fluidos en reservorios de petróleo. Eudeba, pág 20.

- Carlson N, \&t Barnette J. (1988). Determining a reliable estimate of the geothermal gradient from shut-in temperature recordings. SPEAnnual Technical Conference and Exhibition,

- Carslaw HS, \& Jaeger JC. (1959). Conduction of heat in solids.

- Deming D. (1989). Application of bottom-hole temperature corrections in geothermal studies. Geothermics, 18(5-6), pp. 775-786.

- Dowdle WL, \& Cobb WM. (1975). Static formation temperature from well logs-an empirical method. Journal of Petroleum Technology, 27(11), pp. 1326-1330.

- Drury MJ. (1984). On a possible source of error in extracting equilibrium formation temperatures from borehole BHT data. Geothermics, 13(3), pp. 175-180.

- Espinosa-Paredes G, Morales-Diaz A, Olea-González U, \&t Ambriz-Garcia JJ. (2009). Application of a proportionalintegral control for the estimation of static formation temperatures in oil wells. Marine and Petroleum Geology, 26(2), pp. 259-268.

- Hasan AR, \& Kabir CS. (1994). Static reservoir temperature determination from transient data after mud circulation. SPE Drilling and Completion, 9(1), pp 17-24.

- Kritkos WP, \&t Kutasov IM. (1988). Two-point method for determination of undisturbed reservoir temperature. SPE (Society of Petroleum Engineers) Format.Eval, 3(1)

- Roux B, Sanyal S, \& Brown S. (1980). An improved approach to estimating true reservoir temperature from transient temperature data. Fifth Workshop on Geothermal Reservoir Engineering, Stanford, CA, USA. pp 373-384.

- Santoyo E, Garcia A, Espinosa G, Hernandez I, \& Santoyo S. (2000). STATIC_TEMP: A useful computer code for calculating static formation temperatures in geothermal wells. Computers \& Geosciences, 26(2), pp 201-217.

- Shen PY, \& Beck AE. (1986). Stabilization of bottom hole temperature with finite circulation time and fluid flow. Geophysical Journal of the Royal Astronomical Society, 86(1), pp. 63-90.

- Takai K, Hyodo M, \&t Takasugi S. (1994). Estimating of equilibrium formation temperature by curve fitting method and it's problems. NINETEENTH WORKSHOP GEOTHERMAL RESERVOIR ENGINEERING, Stanford, California. 